\title{
Development of an experimental inactivated PRRSV vaccine that induces virus-neutralizing antibodies
}

\author{
Merijn Vanhee, Peter L. Delputte, Iris Delrue, Marc F. Geldhof, \\ Hans J. NAUWYNCK*
}

Laboratory of Virology, Department of Virology, Parasitology and Immunology, Faculty of Veterinary Medicine, Ghent University, Salisburylaan 133, 9820 Merelbeke, Belgium

(Received 16 January 2009; accepted 11 August 2009)

\begin{abstract}
Porcine reproductive and respiratory syndrome virus (PRRSV) can induce reproductive disorders and is involved in the porcine respiratory disease complex, causing tremendous economic losses to the swine industry. Inactivated PRRSV vaccines are preferred over attenuated vaccines because of their safety and flexibility towards emerging virus strains, but the efficacy of current inactivated PRRSV vaccines is questionable. In this study, experimental inactivated PRRSV vaccines were developed, based on two formerly optimized inactivation procedures: UV irradiation and treatment with binary ethylenimine (BEI). In a first experiment, it was shown that vaccination with UV- or BEI-inactivated virus in combination with Incomplete Freund's Adjuvant induced virus-specific antibodies and strongly primed the virus-neutralizing (VN) antibody response. Subsequently, the influence of adjuvants on the immunogenicity of neutralizing epitopes on the inactivated virus was investigated. It was shown that vaccination with BEI-inactivated virus in combination with a commercial oil-in-water adjuvant induced high titers $\left(3.4 \log _{2}\right)$ of VN antibodies in $6 / 6$ pigs, instead of only priming the neutralizing antibody response. After challenge, neutralizing antibody titers in these vaccinated animals rose to a mean value of $5.5 \log _{2}$, and the duration of the viremia was reduced to an average of 1 week. This study shows that, by the use of an optimized inactivation procedure and a suitable adjuvant, inactivated PRRSV vaccines can be developed that induce VN antibodies and offer partial protection upon challenge.
\end{abstract}

PRRSV / inactivated vaccine

\section{INTRODUCTION}

Porcine reproductive and respiratory syndrome virus (PRRSV) is an enveloped positive-stranded RNA virus that belongs to the family of the Arteriviridae in the order of the Nidovirales [9, 25]. PRRSV can cause severe reproductive failure in sows, characterized by late term abortion, early farrowing, stillbirth and the birth of weak piglets, and is associated with the porcine respiratory disease complex in

\footnotetext{
* Corresponding author: hans.nauwynck@ugent.be
}

combination with secondary infections [3, 4, $26-28,30]$. The virus is present in a majority of swine producing countries around the world and gives rise to enormous economic losses in the swine industry [17]. Virus-neutralizing (VN) antibodies against PRRSV protect against viremia, virus replication in lungs, transplacental spreading of the virus and reproductive failure $[12-14,20]$. However, VN antibodies only appear in low amounts around 4 weeks or more after PRRSV infection, which is in contrast with the fast appearance of high amounts of nonneutralizing antibodies [7, 34]. Attenuated as

This is an Open Access article distributed under the terms of the Creative Commons Attribution-Noncommercial License (http://creativecommons.org/licenses/by-nc/3.0/), which permits unrestricted use, distribution, and reproduction in any noncommercial medium, provided the original work is properly cited. 
well as inactivated PRRSV vaccines are frequently used in the field. Attenuated vaccines induce $\mathrm{VN}$ antibodies and protect against viremia, virus replication in lungs and virusinduced respiratory and reproductive disorders $[10,23,35]$. However, the protective immune response induced by attenuated PRRSV vaccines is influenced by genetic diversity, as these vaccines do not always sufficiently protect against virus strains that are genetically different from the vaccine virus strain [11, 15]. Besides, there are major concerns about the safety of attenuated PRRSV vaccines, as the vaccine virus on itself can cause viremia and can spread transplacentally and horizontally with the risk of reverting to virulence [2, 18, 22]. Inactivated vaccines on the other hand are safe and it has been shown by Misinzo et al. that it is possible to stimulate the $\mathrm{VN}$ antibody response in naïve piglets by immunization with inactivated PRRSV [16]. Commercially available inactivated PRRSV vaccines however do not induce VN antibodies and do not sufficiently protect against viremia [19, 24, 35]. $\mathrm{VN}$ antibodies inhibit replication of PRRSV in porcine alveolar macrophages (PAM), the in vivo host cell type for the virus, by blocking attachment to and internalization in the cell [5]. Hence, domains on the virus particle that are involved in binding and internalization are involved in virus neutralization. Delrue et al. [6] recently showed that certain inactivation procedures do not conserve viral entry-associated domains on the virus particle, while others fully do. These findings suggest that the latter inactivation procedures are preferable for the development of inactivated PRRSV vaccines that aim to induce $\mathrm{VN}$ antibodies. In the present study, two inactivation procedures that conserve viral entry-associated domains, namely treatment with ultraviolet (UV) radiation and treatment with binary ethylenimine (BEI), were used in the development of experimental inactivated PRRSV vaccines. The ability of such vaccines to induce a $\mathrm{VN}$ antibody response and protect against viremia after challenge was determined. A commercial inactivated PRRSV vaccine was simultaneously tested to have a reference vaccine available in the used experimental design. Subsequently, the influence of different adjuvants on the immunogenicity of the inactivated virus was determined. After all, an experimental inactivated PRRSV vaccine was developed that induced $\mathrm{VN}$ antibodies and reduced the duration of viremia after infection.

\section{MATERIALS AND METHODS}

\subsection{Virus production and purification}

The PRRSV Lelystad virus (LV) strain, propagated in Marc-145 cells, was used for vaccine preparation [30]. The fifth passage cell culture supernatant was filtrated through a $0.45 \mu \mathrm{m}$ filter and virus was concentrated from the supernatant by ultracentrifugation at $112000 \mathrm{~g}$ for $2 \mathrm{~h}$ using a Type 35 rotor (Beckman Coulter, Analis, Ghent, Belgium). Subsequently, virus was semi-purified by ultracentrifugation at $100000 \mathrm{~g}$ for $3 \mathrm{~h}$ through a $30 \%$ sucrose cushion, using a SW41Ti rotor (Beckman Coulter), resuspended in phosphate buffered saline and stored at $-70{ }^{\circ} \mathrm{C}$. Challenge virus consisted of the fifth passage of PRRSV LV, propagated in PAM that were derived from gnotobiotic piglets.

\subsection{Virus inactivation}

Before inactivation, purified virus was diluted in RPMI 1640 to a titer of $10^{8} \mathrm{TCID}_{50} / \mathrm{mL}$. Inactivation of PRRSV with BEI was performed as described by Bahnemann [1]. A $0.1 \mathrm{M}$ stock of BEI was prepared by cyclization of 2-bromoethylamine in $0.175 \mathrm{M}$ $\mathrm{NaOH}$ for $1 \mathrm{~h}$ at $37^{\circ} \mathrm{C}$ and stored at $4{ }^{\circ} \mathrm{C}$. Virus was inactivated by incubation with $1 \mathrm{mMBEI}$ during $24 \mathrm{~h}$ at $37^{\circ} \mathrm{C}$. Afterwards, BEI was neutralized by incubation with $0.1 \mathrm{mM}$ Na-thiosulphate for $2 \mathrm{~h}$ at $37{ }^{\circ} \mathrm{C}$. Inactivation of PRRSV with UV radiation was performed by irradiation of the virus suspension with $1000 \mathrm{~mJ} / \mathrm{cm}^{2}$, using a UV crosslinker (UVP, Inc., BRS, Drogenbos, Belgium). Inactivated virus was stored at $-70{ }^{\circ} \mathrm{C}$.

\subsection{Analysis of complete inactivation}

To verify if virus was completely inactivated, $1 \mathrm{~mL}$ of each inactivated virus suspension was inoculated on Marc-145 cells in a $150 \mathrm{~cm}^{2}$ tissue culture flask with $50 \mathrm{~mL}$ of medium. The cells were cultivated for 1 week at $37^{\circ} \mathrm{C}$, followed by transfer of the supernatant to a fresh culture and incubation for 
another week. Inoculation of Marc-145 cells with $1 \mathrm{~mL}$ of non-inactivated virus was included as positive control. Cells were analyzed for cytopathic effect (CPE) and all cell cultures were fixed with $100 \%$ methanol at $-20{ }^{\circ} \mathrm{C}$, followed by an immunoperoxidase staining with monoclonal antibody P3/27 against the PRRSV nucleocapsid protein to detect infected cells [31].

\subsection{Analysis of virus internalization in macrophages}

Virus internalization of live and inactivated virus in PAM was performed as described by Delputte et al. [5]. PAM were inoculated with the virus suspensions and incubated for $1 \mathrm{~h}$ at $37{ }^{\circ} \mathrm{C}$ with $5 \%$ $\mathrm{CO}_{2}$. Cells were washed to remove unbound virus particles, fixed with $100 \%$ methanol at $-20{ }^{\circ} \mathrm{C}$, and virus particles were stained with mouse monoclonal antibody P3/27 against the PRRSV nucleocapsid protein and FITC-labelled goat anti-mouse antibody. The amount of internalized virus particles in PAM was determined with a Leica TCS SP2 laser-scanning spectral confocal system (Leica Microsystems, Groot Bijgaarden, Belgium) in five randomly selected cells for each condition. The mean number of internalized virus particles for non-inactivated virus was equalized to $100 \%$ and the relative $\%$ of internalization for inactivated virus was determined.

\subsection{Experimental design of animal studies}

All piglets were derived from a PRRS-negative farm and their PRRSV-negative status was confirmed by immunoperoxidase monolayer assay (IPMA).

\subsubsection{Vaccination with a commercial inactivated vaccine and $U V$ - or BEI-inactivated virus in Incomplete Freund's Adjuvant}

In a first experiment, 24 piglets were randomly assigned to four treatment groups. A first group (group A) served as mock-vaccinated control group and received $1 \mathrm{~mL}$ RPMI in $1 \mathrm{~mL}$ Incomplete Freund's Adjuvant (IFA, Sigma-Aldrich, Bornem, Belgium) at 6 and 10 weeks of age. The other three groups were vaccinated twice intramuscularly at 6 (primo vaccination) and 10 (booster vaccination) weeks of age. Group B was vaccinated with $2 \mathrm{~mL}$ of a commercial European-type inactivated PRRSV vaccine (Progressis ${ }^{\circledR}$, Merial, Brussels, Belgium). Group $\mathrm{C}$ received $1 \mathrm{~mL}$ of $\mathrm{UV}$-inactivated virus in $1 \mathrm{~mL}$ IFA and group $\mathrm{D}$ received $1 \mathrm{~mL}$ of
BEI-inactivated virus in $1 \mathrm{~mL}$ IFA. Four weeks after the booster vaccination, all pigs were challenged by intranasal inoculation of $10^{6} \mathrm{TCID}_{50} \mathrm{LV}$. Blood was taken weekly after vaccination and at $0,3,5$, $7,10,14,21,28$ and 35 days post challenge. Serum was collected and stored at $-70{ }^{\circ} \mathrm{C}$. Serum samples for IPMA and VN antibody detection were incubated during $30 \mathrm{~min}$ at $56{ }^{\circ} \mathrm{C}$ prior to freezing.

\subsubsection{Vaccination with BEI-inactivated virus in Incomplete Freund's Adjuvant or Alhydrogel}

In a second experiment, 18 piglets were randomly assigned to three treatment groups. Group E served as mock-vaccinated control group and received $2 \mathrm{~mL}$ RPMI. The other two groups were vaccinated twice with $1 \mathrm{~mL}$ BEI-inactivated virus in $1 \mathrm{~mL}$ adjuvant. For group F, IFA was used as adjuvant, while for group $\mathrm{G}$, the inactivated virus was diluted in a $16 \%$ aluminium hydroxide colloidal gel (Alhydrogel, Sigma-Aldrich). The same experimental design was used as for the first experiment, but blood was only sampled up till 21 days post challenge.

\subsubsection{Vaccination with BEI-inactivated virus in Alhydrogel or Suvaxyn o/w adjuvant}

In a third experiment, 18 piglets were randomly assigned to three treatment groups. Group H served as mock-vaccinated control group and received $2 \mathrm{~mL}$ RPMI at the time of vaccination. The other two groups were vaccinated twice with $1 \mathrm{~mL}$ BEIinactivated virus in $1 \mathrm{~mL}$ adjuvant. For group I, Alhydrogel was used as adjuvant, while for group $\mathrm{J}$, an oil-in-water $(\mathrm{o} / \mathrm{w})$ diluent that is used in the commercial pseudorabies virus vaccine Suvaxyn ${ }^{\circledR}$ Aujeszky (Fort Dodge Animal Health, Kelmis, Belgium) was used. The same experimental design was used as for the former experiments, and blood was sampled up till 32 days post challenge.

\subsection{Antibody detection and virus titration}

PRRSV-specific serum antibody titers were determined by IPMA as described by Labarque et al. [12]. VN antibody titers were detected by a seroneutralization (SN) test on Marc-145 cells. Briefly, a 1/2 dilution series of serum was prepared and an equal volume of virus with a titer of $2 \times 10^{3} \mathrm{TCID}_{50} / \mathrm{mL}$ was added to each dilution and incubated for $1 \mathrm{~h}$ at $37{ }^{\circ} \mathrm{C}$. Subsequently, the serum-virus mixture was 
transferred to a 96-well plate containing confluent Marc-145 cells, and the cells were analyzed for $\mathrm{CPE}$ at 7 days post inoculation. The $\mathrm{VN}$ antibody titer was defined as the reciprocal of the highest dilution that inhibited CPE in $50 \%$ of the inoculated wells. Virus titers in serum were determined by virus titration on PAM, followed by immunoperoxidase staining with monoclonal antibody P3/27 against the nucleocapsid protein of PRRSV [12].

\subsection{Statistical analysis}

Internalization in macrophages was analyzed by one-way analysis of variance and $p<0.05$ was taken as the level of statistical significance. Antibody and virus titers were analyzed by Kruskall-Wallis test, followed by Dunn's multiple comparisons test to determine differences between groups at different time points. An overall $p$ value of 0.05 was used for this mutiple comparisons test. All statistical analyses were performed using GraphPad Prism version 5.0a (GraphPad Software, San Diego, California, USA).

\section{RESULTS}

\subsection{Virus inactivation and control of internalization}

For each vaccination experiment, a PRRSV virus suspension with a titer of $10^{8} \mathrm{TCID}_{50} /$ $\mathrm{mL}$ was inactivated with either UV radiation or BEI. Complete inactivation was evaluated by passage of a sample on Marc-145 cells, followed by immunostaining for the nucleocapsid protein of PRRSV to detect infected cells. Cells that were inoculated with inactivated virus never showed any CPE or positive nucleocapsid staining, while a clear CPE as well as nucleocapsid staining was observed on cell cultures that were inoculated with non-inactivated virus. To determine whether inactivation had influenced the capacity of the virus to get internalized in PAM, an internalization experiment was performed with BEI- or UV-inactivated virus and a non-inactivated virus suspension as positive control. The relative percentage of internalized virus particles per cell did not differ significantly $(p=0.08)$ between UV-inactivated $(86 \pm 11)$, BEI-inactivated $(131 \pm 15)$ and not inactivated virus $(100 \pm 13)$.

\subsection{Vaccination with a commercial inactivated vaccine and $U V$ - or BEI-inactivated virus in Incomplete Freund's Adjuvant}

\subsubsection{Virus-specific and -neutralizing antibodies}

PRRSV-specific antibodies were not detected by IPMA in any of the pigs of the adjuvant control group (group A) before challenge (Fig. 1, upper panel). Of pigs that were vaccinated with the commercial inactivated vaccine (group B), one pig transiently showed a positive IPMA antibody titer after vaccination and another had seroconverted at the day of challenge, while the other pigs remained seronegative up till the time of challenge. In contrast, all pigs that were vaccinated with the experimental UV-inactivated (group C) or BEI-inactivated (group D) vaccine already showed positive antibody titers at 3 weeks after the primo vaccination. After the booster vaccination antibody titers rose to values as high as normally seen after PRRSV infection in naïve pigs [12]. After challenge, all pigs had seroconverted at day 10. Antibody titers in group B were slightly higher than in group $\mathrm{A}$, however differences were not significant. In group $\mathrm{C}$ as well as group D, antibody titers were significantly higher compared to group A starting from 1 week after booster vaccination up till 1 week post challenge.

Before challenge, none of the pigs showed $\mathrm{VN}$ antibodies, except for one pig of group $\mathrm{D}$ that showed an SN antibody titer of $1.0 \log _{2}$ at 2 weeks after the booster vaccination and at the day of challenge (Fig. 1, lower panel). After challenge, one pig of group A showed a transient SN antibody titer at day 10 , however for all other adjuvant control pigs, VN antibodies only appeared between 3 and 5 weeks post challenge and one pig even remained negative during the entire experiment. Only four pigs of group B showed VN antibodies after challenge, two starting from 10 days, one at 4 weeks and another at 5 weeks post challenge, and VN antibody titers did not differ between group A and B at any time point. In contrast, all pigs of group $\mathrm{C}$ and $\mathrm{D}$ showed $\mathrm{VN}$ antibodies after challenge. In group $\mathrm{C}$, five animals seroconverted for $\mathrm{VN}$ antibodies within the first 2 weeks after challenge, and the 

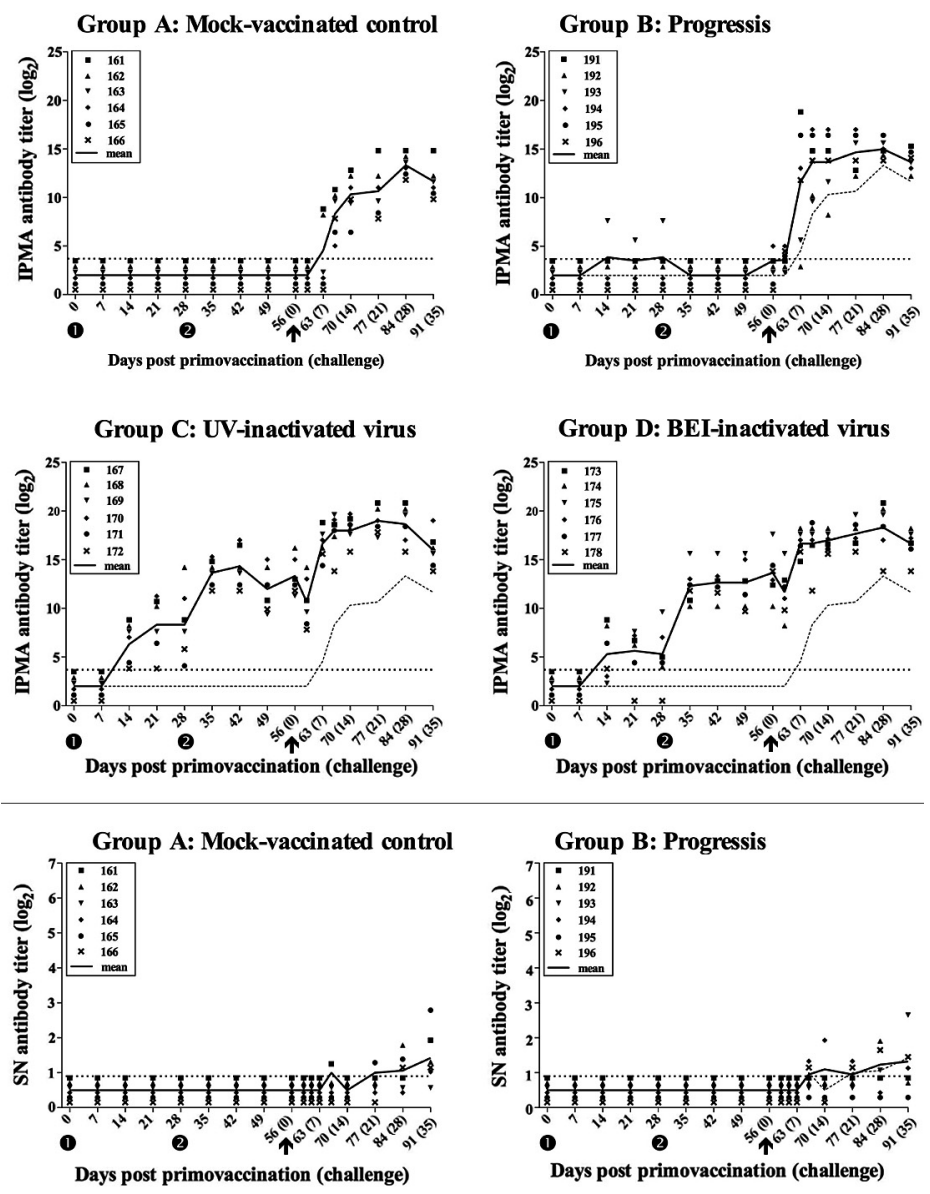

Group C: UV-inactivated virus

Group D: BEI-inactivated virus
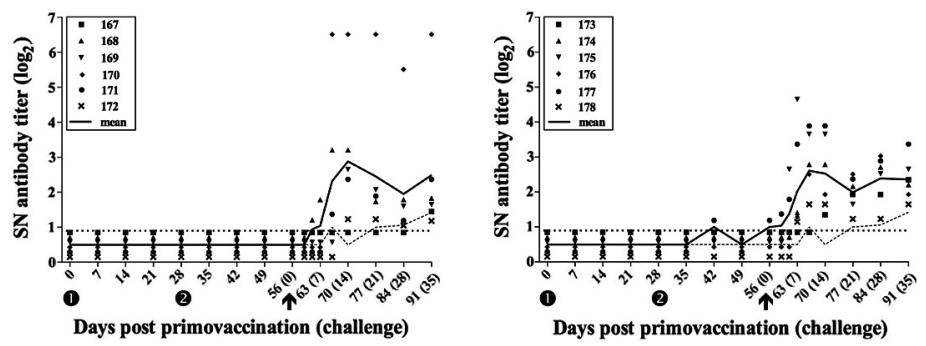

Figure 1. PRRSV-specific IPMA antibody titers $\left(\log _{2}\right)$ (upper panel) and VN antibody titers $\left(\log _{2}\right)$ (lower panel) after vaccination and challenge for group A (Mock-vaccinated control), B (Progressis), C (UVinactivated virus) and D (BEI-inactivated virus). $\mathbf{0}=$ primo vaccination, $\mathbf{0}=$ booster vaccination and $\uparrow=$ challenge. Symbols represent individual animals and lines represent mean IPMA or SN titers for each group. The dashed line gives the mean titers for group A. The dotted line gives the detection limit for the IPMA or SN test. 
sixth one after 5 weeks. VN antibody titers were significantly higher in group $\mathrm{C}$ compared to group $\mathrm{A}$ at 2 weeks post challenge, reaching mean values of $2.9 \log _{2}$. In group $\mathrm{D}$, one pig already had $\mathrm{VN}$ antibodies before challenge and starting from 1 week post challenge all pigs of this group showed a positive $\mathrm{SN}$ antibody titer, except for one animal that became positive 1 week later. $\mathrm{VN}$ antibody titers were significantly higher in group D compared to group A at 10, 14 and 21 days post challenge, reaching mean values of 2.6, 2.5 and $2.0 \log _{2}$ respectively.

\subsubsection{Viremia}

Virus was not detected in serum at 1 week after each immunization and at the day of challenge, confirming that all vaccines were properly inactivated. After challenge, all animals showed viremia. Figure 2 represents virus titers $\left(\log _{10}\right.$ $\mathrm{TCID}_{50} / \mathrm{mL}$ ) post challenge for each group. In group $\mathrm{A}$, a maximum mean virus titer of 3.5 $\log _{10} \mathrm{TCID} \mathrm{D}_{50} / \mathrm{mL}$ was reached at day 5 post challenge and a second peak of $3.1 \log _{10} \mathrm{TCID}_{50} / \mathrm{mL}$ was observed at day 10 . Subsequently, a decline in virus titers was observed, and virus could no longer be detected at 2, 3 or 4 weeks after challenge (two animals per time point). Mean virus titers in group B were slightly lower compared to those in group A, being $3.1 \log _{10} \mathrm{TCID}_{50} / \mathrm{mL}$ at 5 days and $2.0 \log _{10} \mathrm{TCID}_{50} / \mathrm{mL}$ at 10 days post infection, but no significant differences could be detected at any time point between group A and group B. In one animal of this group, virus was cleared from the blood at 1 week post challenge, in another one at 10 days and in the other animals at 2, 3 or 4 weeks. The mean virus titer for group $\mathrm{C}$ was $2.7 \log _{10} \mathrm{TCID}_{50} / \mathrm{mL}$ at 5 days and $2.5 \log _{10} \mathrm{TCID}_{50} / \mathrm{mL}$ at 10 days post challenge, however titers were not significantly different from those in group $\mathrm{A}$ at any time point. Nevertheless, viremia in group $\mathrm{C}$ was already cleared at 2 weeks post challenge for five animals, and at 3 weeks for the sixth one. Finally, the mean virus titer for group D was $2.6 \log _{10}$ $\mathrm{TCID}_{50} / \mathrm{mL}$ at 5 days post challenge and 1.4 $\log _{10} \mathrm{TCID}_{50} / \mathrm{mL}$ at day 10 , and at the latter time point virus titers were significantly reduced, compared to group A. Viral clearance was observed for one animal per time point at 7, 10,
14 and 21 days and for two animals at 28 days post challenge.

\subsection{Vaccination with BEI-inactivated virus in Incomplete Freund's Adjuvant or Alhydrogel}

\subsubsection{Virus-specific and -neutralizing antibodies}

All mock-vaccinated animals (group E) showed virus-specific antibodies starting from 10 days post challenge (Fig. 3, upper panel). Animals that were vaccinated with BEIinactivated virus in combination with IFA adjuvant (group F) seroconverted at 2 or 3 weeks after the first vaccination. Two animals that were vaccinated with BEI-inactivated virus in combination with Alhydrogel as adjuvant (group G) showed virus-specific antibodies from 2 weeks after the first vaccination, and the other animals in this group starting from 2 weeks after booster vaccination. Antibody titers in both vaccinated groups were significantly higher compared to the control group from 2 weeks after booster vaccination up till 10 days post challenge, and antibody titers in group $\mathrm{F}$ were generally higher compared to group $\mathrm{G}$.

VN antibodies could not be detected in group $\mathrm{E}$ at any time point, except for one animal at 2 weeks post challenge (Fig. 3, lower panel). None of the vaccinated animals showed VN antibodies before challenge, but VN antibodies already appeared between 5 and 10 days after challenge in group F, and between 7 and 10 days after challenge in group G. VN antibody titers were significantly higher in both vaccinated groups, compared to the control group starting from 10 days post challenge up till the end of the study. Mean VN antibody titers were in general $1 \log _{2}$ higher in group $\mathrm{F}$ than in group $\mathrm{G}$, reaching a maximum of 4.0 $\log _{2}$ at 2 weeks post challenge.

\subsubsection{Viremia}

All animals in group E showed viremia after challenge, and a peak mean virus titer of 3.3 $\log _{10} \mathrm{TCID}_{50} / \mathrm{mL}$ was observed at day 5 (Fig. 4). Viral clearance was observed for one animal at 2 weeks, and for another at 3 weeks 


\section{Group A: Mock-vaccinated control}

\section{Group B: Progressis}
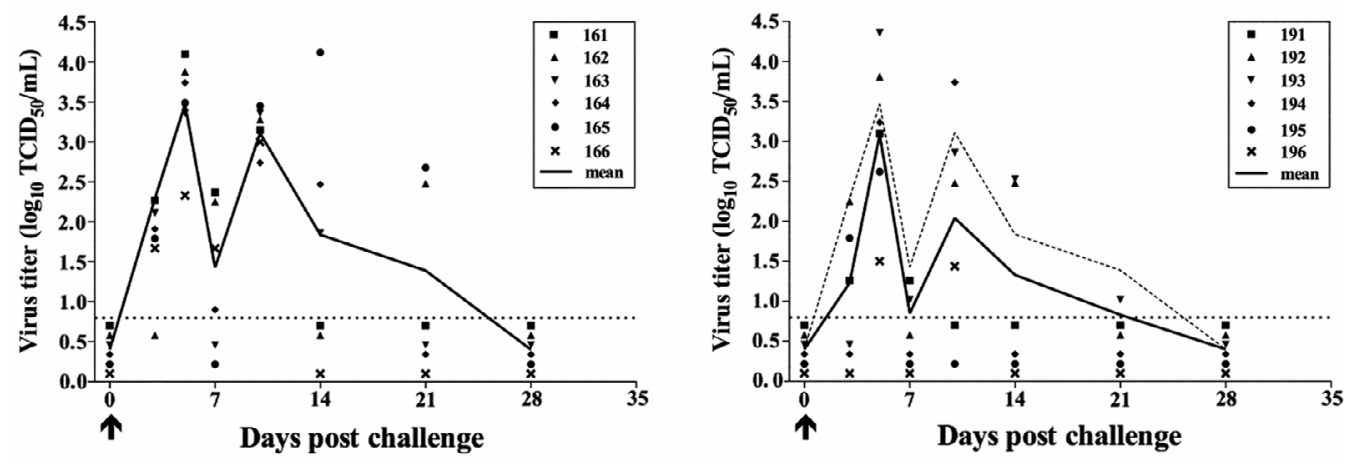

Group C: UV-inactivated virus

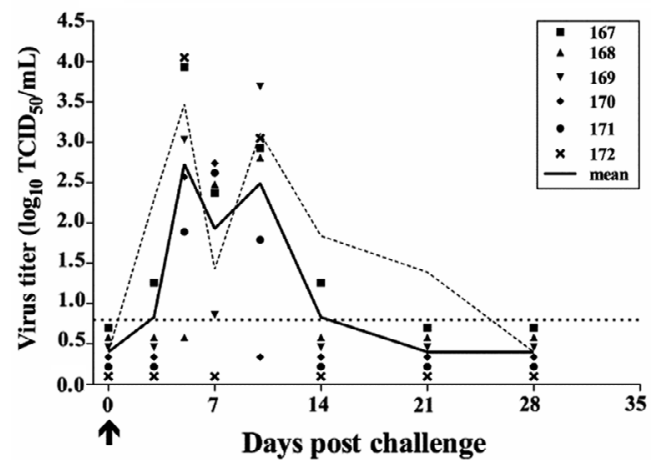

Group D: BEI-inactivated virus

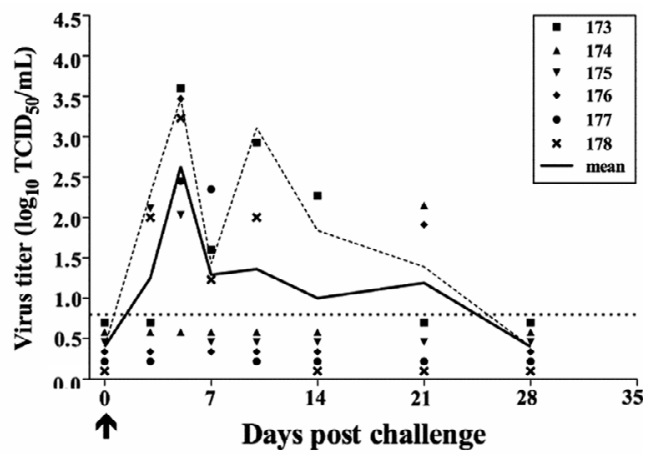

Figure 2. Serum-virus titers after challenge for group A (Mock-vaccinated control), B (Progressis), C (UVinactivated virus) and D (BEI-inactivated virus). Virus titers in serum $\left(\log _{10} \mathrm{TCID}_{50} / \mathrm{mL}\right)$ were determined by virus titration on PAM, followed by immunoperoxidase staining for the PRRSV nucleocapsid protein. $\uparrow=$ challenge. Symbols represent individual animals and lines represent mean virus titers in each group. The dashed line gives the mean titers for group A. The dotted line gives the detection limit for virus titration.

after challenge. The other animals in this group stayed viremic till the end of the experiment (3 weeks post challenge). In group $\mathrm{F}$, one animal did not show any viremia at all. The mean peak viremia in group $\mathrm{F}$ was reduced to 2.4 $\log _{10} \mathrm{TCID}_{50} / \mathrm{mL}$ at 5 days post challenge and to $1.0 \log _{10} \mathrm{TCID}_{50} / \mathrm{mL}$ at day 7 , compared to group E, and at the latter time point, this reduction was significant. For animals that showed viremia, virus was cleared from the blood for each one animal at day 5, 7 or 10 , and at day 14 for the two others. In group $\mathrm{G}$, the peak viremia at day 5 was not reduced, but virus titers at day 7 were significantly reduced to $1.1 \log _{10} \mathrm{TCID}_{50} / \mathrm{mL}$. Virus could no longer be detected from 7 days post challenge for half of the animals. The other animals in this group were virus-negative at 2 weeks post challenge, but two of them again showed a positive virus titer at 3 weeks.

\subsection{Vaccination with BEI-inactivated virus in Alhydrogel or Suvaxyn o/w adjuvant}

\subsubsection{Virus-specific and -neutralizing antibodies}

One animal in the mock-vaccinated group (group $\mathrm{H}$ ) seroconverted at 7 days post 


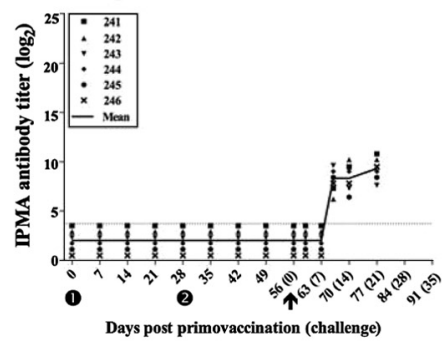

\section{Group F: IFA adjuvant}
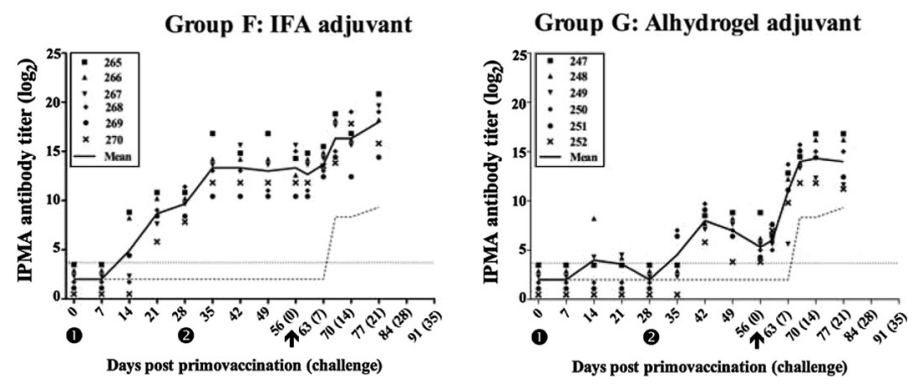

Group E: Mock-vaccinated control

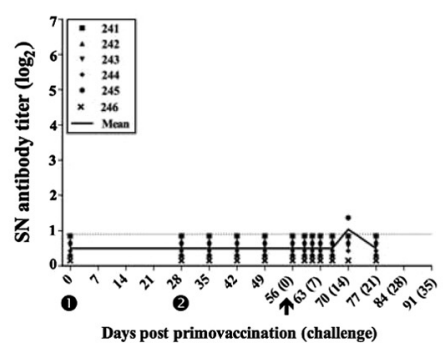

Group F: IFA adjuvant

Group G: Alhydrogel adjuvant
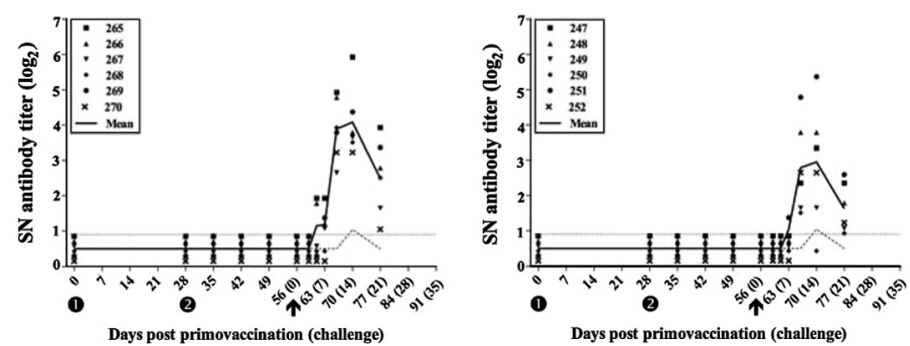

Figure 3. PRRSV-specific IPMA antibody titers $\left(\log _{2}\right)$ (upper panel) and VN antibody titers $\left(\log _{2}\right)$ (lower panel) after vaccination and challenge for group E (Mock-vaccinated control), F (IFA adjuvant) and G (Alhdyrogel adjuvant) $\boldsymbol{0}=$ primo vaccination, $\mathbf{2}=$ booster vaccination and $\boldsymbol{\uparrow}=$ challenge. Symbols represent individual animals and lines represent mean IPMA or SN titers for each group. The dashed line gives the mean titers for group E. The dotted line gives the detection limit for the IPMA or SN test. 


\section{Group E: Mock-vaccinated control}

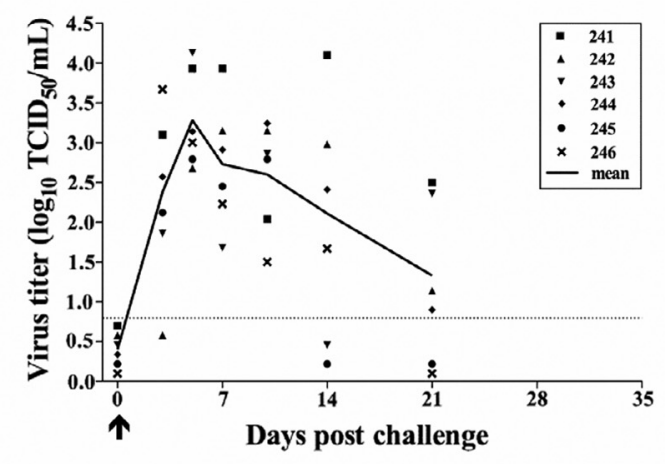

\section{Group F: IFA adjuvant}

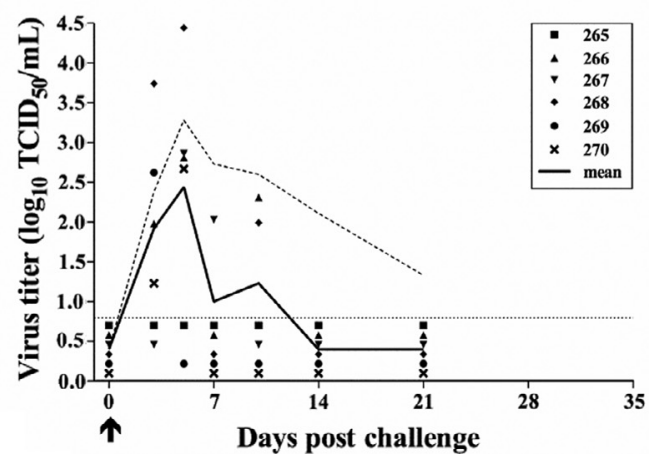

\section{Group G: Alhydrogel adjuvant}

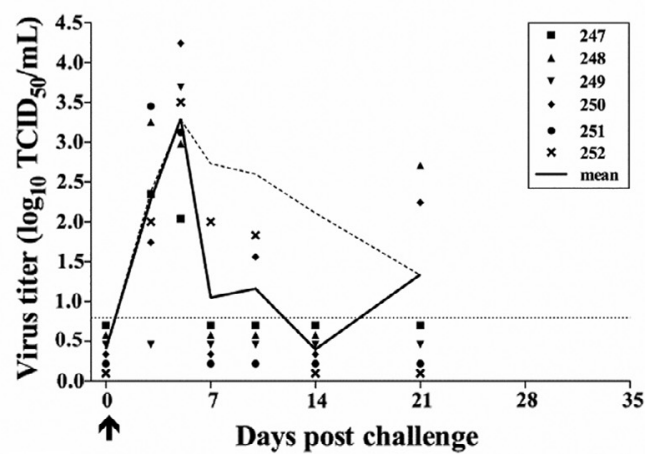

Figure 4. Serum-virus titers after challenge for group E (Mock-vaccinated control), F (IFA adjuvant) and G (Alhydrogel adjuvant). Virus titers in serum $\left(\log _{10} \mathrm{TCID}_{50} / \mathrm{mL}\right)$ were determined by virus titration on PAM, followed by immunoperoxidase staining for the PRRSV nucleocapsid protein. $\uparrow=$ challenge. Symbols represent individual animals and lines represent mean virus titers in each group. The dashed line gives the mean titers for group E. The dotted line gives the detection limit for virus titration.

challenge, and the others at 10 days (Fig. 5, upper panel). Five animals that were vaccinated with BEI-inactivated virus in combination with Alhydrogel adjuvant (group I) showed virusspecific antibodies from 3 weeks after the first vaccination, and one animal from 1 week after booster vaccination. Antibody titers in group I were significantly higher than in group $\mathrm{H}$ at 1 week post challenge. In the group that was vaccinated with BEI-inactivated virus in combination with Suvaxyn o/w adjuvant (group J), three animals seroconverted at 3 weeks after the first vaccination, and the others at 1 week after booster vaccination. Antibody titers in group $\mathrm{J}$ were significantly higher than in group $\mathrm{H}$ at 1 and 2 weeks after booster vaccination, and at 1 week post challenge. Antibody titers in both vaccinated groups reached similar values after challenge.

VN antibodies could not be detected in group $\mathrm{H}$ earlier than 3 weeks after challenge, and it lasted more than 4 weeks before all animals in this group had shown VN antibodies (Fig. 5, lower panel). In group I, no animals showed VN antibodies before challenge, but all animals seroconverted for $\mathrm{VN}$ antibodies between one and 2 weeks post challenge, and at 2 weeks post challenge, titers were 
Group H: Mock-vaccinated control

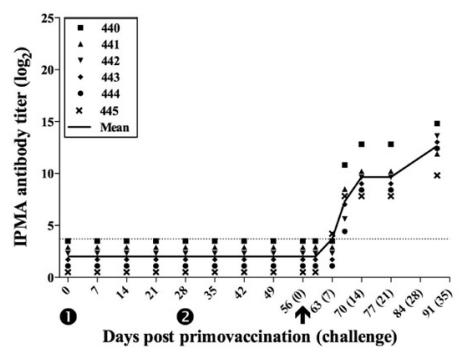

Group I: Alhydrogel adjuvant

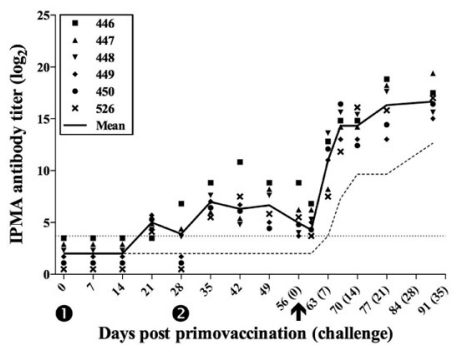

Group J: Suvaxyn o/w adjuvant

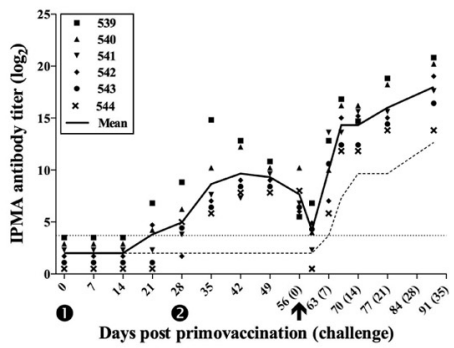

Group H: Mock-vaccinated control

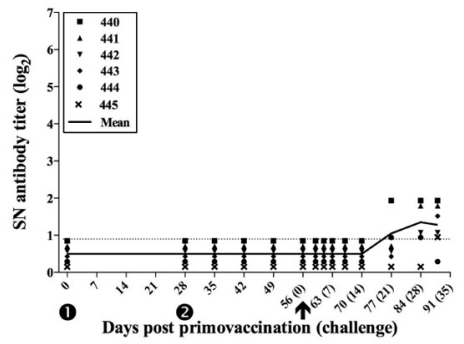

Group I: Alhydrogel adjuvant

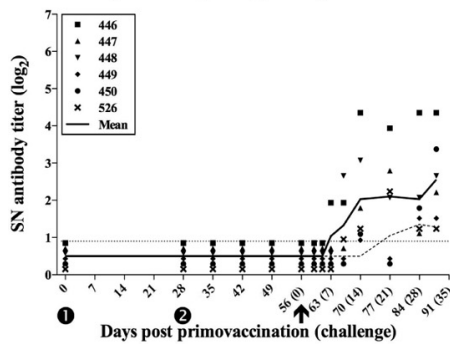

Group J: Suvaxyn o/w adjuvant

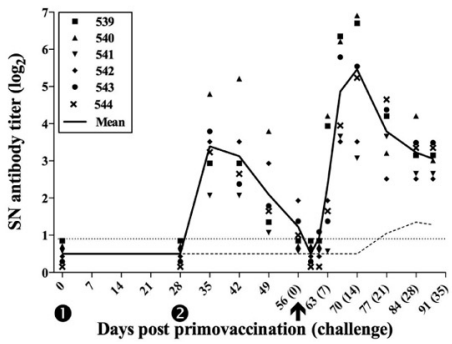

Figure 5. PRRSV-specific IPMA antibody titers $\left(\log _{2}\right)$ (upper panel) and VN antibody titers $\left(\log _{2}\right)$ (lower panel) after vaccination and challenge for group $\mathrm{H}$ (Mock-vaccinated control), I (Alhydrogel adjuvant) and J (Suvaxyn $\mathrm{o} / \mathrm{w}$ adjuvant) $\boldsymbol{0}=$ primo vaccination, $\boldsymbol{\theta}=$ booster vaccination and $\boldsymbol{\uparrow}=$ challenge. Symbols represent individual animals and lines represent mean IPMA or SN titers for each group. The dashed line gives the mean titers for group $\mathrm{H}$. The dotted line gives the detection limit for the IPMA or SN test. 
significantly different compared to group $\mathrm{H}$ (2.0 $\log _{2}$ ). In group $\mathrm{J}$, however, all animals showed significantly high titers of $\mathrm{VN}$ antibodies already at $1\left(3.4 \log _{2}\right)$ and $2\left(3.1 \log _{2}\right)$ weeks after booster vaccination. Starting from 3 weeks after booster vaccination, $\mathrm{VN}$ antibody titers declined, but they increased again after challenge to a maximum value of $5.5 \log _{2}$ and stabilized to $3.1 \log _{2}$ at the end of the experiment.

\subsubsection{Viremia}

All animals in this experiment showed viremia after challenge (Fig. 6). The mean peak virus titer in group $\mathrm{H}$ was observed at day 5 and reached $2.6 \log _{10} \mathrm{TCID}_{50} / \mathrm{mL}$. Virus was cleared from the blood at 10 days post challenge for one animal, at 2 or 3 weeks for each two animals and at 4 weeks for the sixth animal. No significant reduction in virus titers could be observed in group I, but viral clearance was already observed at day 7 (one animal), 10 (two animals) or 14 (three animals) after challenge. In group J, the peak virus titer was not reduced, but four animals already showed viral clearance at 1 week post challenge, and a fifth one at 2 weeks. The remaining animal was virus-negative at 10 days post challenge, but then again showed a high virus titer at 2 weeks, followed by clearance of viremia at 3 weeks post challenge.

\section{DISCUSSION}

Attenuated as well as inactivated vaccines are currently used for the control of PRRSV in the field. Taking into account both safety and flexibility towards emerging virus strains, inactivated vaccines are preferred over attenuated vaccines, but in spite of these benefits, the efficacy of current inactivated PRRSV vaccines is questionable [24, 35]. In the current study, experimental inactivated PRRSV vaccines were developed, based on formerly optimized inactivation procedures, and the efficacy of these vaccines was evaluated. As a reference, the efficacy of a commercial inactivated PRRSV vaccine was simultaneously tested in the first experiment. This vaccine did not induce virus-specific antibodies, and only slightly elevated the antibody response after challenge. These results are similar to a study, performed by Zuckermann et al. [35], showing that vaccination with this commercial vaccine only resulted in an anamnestic humoral immune response after challenge. Virus-specific antibody titers as measured by IPMA or ELISA do not correlate with $\mathrm{VN}$ antibody titers and hence do not provide any information about protective immunity against PRRSV [33]. In the present experiment, the $\mathrm{VN}$ antibody response after infection in mock-vaccinated animals was delayed and weak, compared to the virus-specific antibody response. This was not surprising, as it has been described that the VN antibody response against PRRSV is strongly impaired [7, 12, 34]. Vaccination with the commercial inactivated PRRSV vaccine did not influence the $\mathrm{VN}$ antibody response after challenge. This is in contrast with the study from Zuckermann et al. [35] that showed an anamnestic $\mathrm{VN}$ antibody response in pigs that were vaccinated with this vaccine, and a study from Scortti et al. [24] where vaccination with another inactivated PRRSV vaccine slightly improved the VN antibody response after challenge. This discrepancy may be due to the use of other tests with different sensitivity for the detection of $\mathrm{VN}$ antibodies [12, 32, 33, 35]. Delrue et al. [6] previously showed that inactivation of PRRSV with UV radiation or BEI does not influence the capacity of the inactivated virus to get internalized in PAM, the in vivo host cell type for the virus. As VN antibodies block internalization of PRRSV in PAM, the degree of internalization might be a measure for conservation of neutralizing antigens after inactivation [5]. In the current study, vaccination with both UV- or BEI-inactivated virus with IFA as adjuvant strongly induced virusspecific antibodies in all animals, and resulted in an earlier and strongly elevated $\mathrm{VN}$ antibody response after infection. The strong priming of the $\mathrm{VN}$ antibody response by vaccination with UV- or BEI-inactivated virus suggests that intact neutralizing antigens were present on the vaccine virus. Although the $\mathrm{VN}$ antibody response was primed by vaccination with 

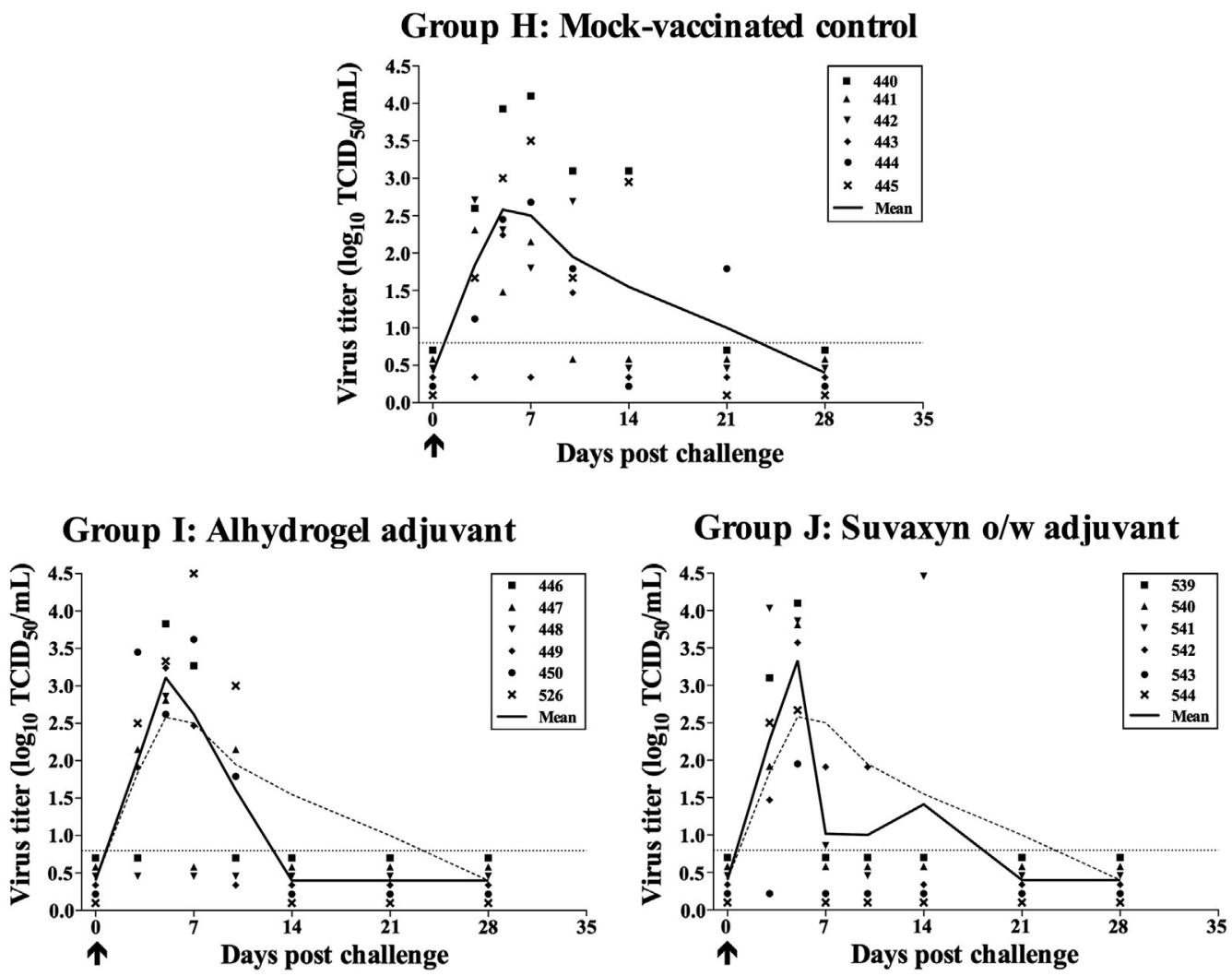

Figure 6. Serum-virus titers after challenge for group H (Mock-vaccinated control), I (Alhydrogel adjuvant) and $\mathrm{J}$ (Suvaxyn o/w adjuvant). Virus titers in serum $\left(\log _{10} \mathrm{TCID}{ }_{50} / \mathrm{mL}\right)$ were determined by virus titration on PAM, followed by immunoperoxidase staining for the PRRSV nucleocapsid protein. $\uparrow=$ challenge. Symbols represent individual animals and lines represent mean virus titers in each group. The dashed line gives the mean titers for group H. The dotted line gives the detection limit for virus titration.

UV- or BEI-inactivated virus, no VN antibodies could be induced by vaccination on itself in the first two experiments, except for one pig that was vaccinated with BEI-inactivated virus in combination with IFA. The low immunogenicity of neutralizing epitopes is characteristic for the virus in itself, as the VN antibody response after PRRSV infection is strongly impaired [7, 12, 34]. To determine to what extent the antibody response, induced by the inactivated virus, was subject to the choice of adjuvant, two different adjuvants that are suitable for field use were tested in combination with the BEI-inactivated virus. Vaccination in combination with Alhydrogel, a commonly used adjuvant in human and veterinary medicine, also consistently induced virus-specific antibodies, and primed the VN antibody response. Nonetheless, both virus-specific and neutralizing antibodies appeared later after vaccination or challenge and titers were lower if Alhydrogel was used instead of IFA. The use of a commercial o/w adjuvant for the BEI-inactivated virus resulted in a comparable IPMA antibody response as with Alhydrogel. Surprisingly, vaccination in combination with the $\mathrm{o} / \mathrm{w}$ adjuvant not only primed the VN antibody response, but also induced high titers of $\mathrm{VN}$ antibodies before challenge. In addition, VN antibodies after challenge rose to titers that were 
higher than those observed by vaccination with IFA or Alhydrogel as adjuvant. The fact that VN antibodies could be induced by vaccination on itself directly proves that neutralizing epitopes are conserved on the BEI-inactivated virion, but the immunogenicity of these epitopes seems subject to the choice of adjuvant. In general, this is the first report of an inactivated PRRSV vaccine that manages to induce a strong VN antibody response by vaccination on itself.

VN antibodies can completely block PRRSV infection of PAM in vitro [5], but the role of these antibodies for in vivo protection against PRRSV is a matter of debate (reviewed in [13]). It has been shown that viremia after infection can be cleared in the absence of detectable levels of $\mathrm{VN}$ antibodies in serum and otherwise viremia can sometimes persist despite the presence of VN antibodies [8, 29]. On the other hand, Labarque et al. showed that clearance of virus from lungs and serum coincides with the appearance of VN antibodies in serum and broncho-alveolar lavage fluid [10]. Moreover, experiments where passive transfer of $\mathrm{VN}$ antibodies was performed before infection with PRRSV showed that these antibodies are able to fully protect pigs against viremia and reproductive failure $[14,20]$. These data indicate that although other mechanisms may be involved in protection against PRRSV, the presence of sufficient amounts of VN antibodies contributes to clearance of viremia, can protect against reproductive failure and even can be sufficient to prevent infection. In the present study, virus was cleared from the blood in mock-vaccinated animals between 2 and 4 weeks post infection when almost no $\mathrm{VN}$ antibodies were present, indicating that viral clearance in those animals took place independent of $\mathrm{VN}$ antibodies. Animals that were vaccinated with the commercial inactivated PRRSV vaccine also did not consistently develop VN antibodies post infection, although a slight reduction in viremia was observed compared to control animals. Piras et al. recently showed that this vaccine can induce a virus-specific interferon- $\gamma(\mathrm{IFN}-\gamma)$ response that may contribute to viral clearance [21]. Another study however showed that a majority of the IFN- $\gamma$ response induced by this vaccine is not PRRSV-specific and is probably induced by a non-viral component of the vaccine [35]. All experimental vaccines tested in the current study were able to reduce viremia, starting from 1 week post infection. A significant reduction in virus titers could be observed in animals that were vaccinated with UV- or BEI-inactivated virus, the latter in combination with both IFA and Alhydrogel, at 7 or 10 days post infection. Moreover, viral clearance was systematically observed at earlier time points in vaccinated animals, compared to control animals, and reduction and/or clearance of viremia always coincided with the appearance of VN antibodies. The use of different adjuvants affected the efficacy of the experimental vaccine regarding reduction of viremia, analogous to the induction of $\mathrm{VN}$ antibodies. However, in spite of the strong induction of $\mathrm{VN}$ antibodies by BEI-inactivated virus in combination with the o/w adjuvant, viremia was still present during the first 5 days after infection in all animals. Remarkably, $\mathrm{VN}$ antibodies were hardly present during this time period, but once they appeared again, virus was cleared in all but one animal. Probably, higher titers of $\mathrm{VN}$ antibodies are needed at the time of challenge to offer full protection against the high dose of virus used to infect the animals. In this study, an experimental inactivated PRRSV vaccine was developed by the use of a formerly optimized inactivation procedure and a suitable adjuvant. This vaccine was able to induce $\mathrm{VN}$ antibodies, and reduced the duration of viremia after infection to 1 week. This is the first report of an inactivated PRRSV vaccine that manages to induce $\mathrm{VN}$ antibodies, which offers new perspectives for the development of effective and safe PRRSV vaccines.

Acknowledgements. The authors want to acknowledge Chantal Vanmaercke, Chris Bracke, Geert Opsomer and Fernand De Backer for excellent technical assistance. Jan Van Doorsselaere and Dries Helderweirt are acknowledged for virus production and purification. This work was funded by the Agricultural Research Programme from the Institute for the Promotion of Innovation by Science and Technology in Flanders. P.L. Delputte is a post-doctoral fellow of the Fund for Scientific Research Flanders (FWO). All animal experiments were approved by the local ethical 
committee of the Faculty of Veterinary Medicine, Ghent University.

\section{REFERENCES}

[1] Bahnemann H.G., Inactivation of viral antigens for vaccine preparation with particular reference to the application of binary ethylenimine, Vaccine (1990) 8:299-303.

[2] Botner A., Strandbygaard B., Sorensen K.J., Have P., Madsen K.G., Madsen E.S., Alexandersen S., Appearance of acute PRRS-like symptoms in sow herds after vaccination with a modified live PRRS vaccine, Vet. Rec. (1997) 141:497-499.

[3] Christianson W.T., Collins J.E., Benfield D.A., Harris L., Gorcyca D.E., Chladek D.W., et al., Experimental reproduction of swine infertility and respiratory syndrome in pregnant sows, Am. J. Vet. Res. (1992) 53:485-488.

[4] Collins J.E., Benfield D.A., Christianson W.T., Harris L., Hennings J.C., Shaw D.P., et al., Isolation of swine infertility and respiratory syndrome virus (isolate ATCC VR-2332) in North America and experimental reproduction of the disease in gnotobiotic pigs, J. Vet. Diagn. Invest. (1992) 4:117-126.

[5] Delputte P.L., Meerts P., Costers S., Nauwynck H.J., Effect of virus-specific antibodies on attachment, internalization and infection of porcine reproductive and respiratory syndrome virus in primary macrophages, Vet. Immunol. Immunopathol. (2004) 102:179-188.

[6] Delrue I., Delputte P.L., Nauwynck H.J., Assessing the functionality of viral entry-associated domains of porcine reproductive and respiratory syndrome virus during inactivation procedures, a potential tool to optimize inactivated vaccines, Vet. Res. (2009) 40:62.

[7] Diaz I., Darwich L., Pappaterra G., Pujols J., Mateu E., Immune responses of pigs after experimental infection with a European strain of Porcine reproductive and respiratory syndrome virus, J. Gen. Virol. (2005) 86:1943-1951.

[8] Diaz I., Darwich L., Pappaterra G., Pujols J., Mateu E., Different European-type vaccines against porcine reproductive and respiratory syndrome virus have different immunological properties and confer different protection to pigs, Virology (2006) 351: 249-259.

[9] Gorbalenya A.E., Enjuanes L., Ziebuhr J., Snijder E.J., Nidovirales: evolving the largest RNA virus genome, Virus Res. (2006) 117:17-37.
[10] Labarque G., Van Gucht S., Van Reeth K., Nauwynck H., Pensaert M., Respiratory tract protection upon challenge of pigs vaccinated with attenuated porcine reproductive and respiratory syndrome virus vaccines, Vet. Microbiol. (2003) 95:187-197.

[11] Labarque G., Reeth K.V., Nauwynck H., Drexler C., Van Gucht S., Pensaert M., Impact of genetic diversity of European-type porcine reproductive and respiratory syndrome virus strains on vaccine efficacy, Vaccine (2004) 22:4183-4190.

[12] Labarque G.G., Nauwynck H.J., Van Reeth K., Pensaert M.B., Effect of cellular changes and onset of humoral immunity on the replication of porcine reproductive and respiratory syndrome virus in the lungs of pigs, J. Gen. Virol. (2000) 81:1327-1334.

[13] Lopez O.J., Osorio F.A., Role of neutralizing antibodies in PRRSV protective immunity, Vet. Immunol. Immunopathol. (2004) 102:155-163.

[14] Lopez O.J., Oliveira M.F., Garcia E.A., Kwon B.J., Doster A., Osorio F.A., Protection against porcine reproductive and respiratory syndrome virus (PRRSV) infection through passive transfer of PRRSV-neutralizing antibodies is dose dependent, Clin. Vaccine Immunol. (2007) 14:269-275.

[15] Mengeling W.L., Lager K.M., Vorwald A.C., Koehler K.J., Strain specificity of the immune response of pigs following vaccination with various strains of porcine reproductive and respiratory syndrome virus, Vet. Microbiol. (2003) 93:13-24.

[16] Misinzo G., Delputte P.L., Meerts P., Drexler C., Nauwynck H.J., Efficacy of an inactivated PRRSV vaccine: induction of virus-neutralizing antibodies and partial virological protection upon challenge, Adv. Exp. Med. Biol. (2006) 581:449-454.

[17] Neumann E.J., Kliebenstein J.B., Johnson C.D., Mabry J.W., Bush E.J., Seitzinger A.H., et al., Assessment of the economic impact of porcine reproductive and respiratory syndrome on swine production in the United States, J. Am. Vet. Med. Assoc. (2005) 227:385-392.

[18] Nielsen H.S., Oleksiewicz M.B., Forsberg R., Stadejek T., Botner A., Storgaard T., Reversion of a live porcine reproductive and respiratory syndrome virus vaccine investigated by parallel mutations, J. Gen. Virol. (2001) 82:1263-1272.

[19] Nilubol D., Platt K.B., Halbur P.G., Torremorell M., Harris D.L., The effect of a killed porcine reproductive and respiratory syndrome virus (PRRSV) vaccine treatment on virus shedding in previously PRRSV infected pigs, Vet. Microbiol. (2004) 102: $11-18$.

[20] Osorio F.A., Galeota J.A., Nelson E., Brodersen B., Doster A., Wills R., et al., Passive transfer of 
virus-specific antibodies confers protection against reproductive failure induced by a virulent strain of porcine reproductive and respiratory syndrome virus and establishes sterilizing immunity, Virology (2002) 302:9-20.

[21] Piras F., Bollard S., Laval F., Joisel F., Reynaud G., Charreyre C., et al., Porcine reproductive and respiratory syndrome (PRRS) virus-specific interferongamma(+) T-cell responses after PRRS virus infection or vaccination with an inactivated PRRS vaccine, Viral Immunol. (2005) 18:381-389.

[22] Scortti M., Prieto C., Martinez-Lobo F.J., Simarro I., Castro J.M., Effects of two commercial European modified-live vaccines against porcine reproductive and respiratory syndrome viruses in pregnant gilts, Vet. J. (2006) 172:506-514.

[23] Scortti M., Prieto C., Simarro I., Castro J.M., Reproductive performance of gilts following vaccination and subsequent heterologous challenge with European strains of porcine reproductive and respiratory syndrome virus, Theriogenology (2006) 66: 1884-1893.

[24] Scortti M., Prieto C., Alvarez E., Simarro I., Castro J.M., Failure of an inactivated vaccine against porcine reproductive, respiratory syndrome to protect gilts against a heterologous challenge with PRRSV, Vet. Rec. (2007) 161:809-813.

[25] Snijder E.J., Meulenberg J.J., The molecular biology of arteriviruses, J. Gen. Virol. (1998) 79: 961-979.

[26] Terpstra C., Wensvoort G., Pol J.M., Experimental reproduction of porcine epidemic abortion and respiratory syndrome (mystery swine disease) by infection with Lelystad virus: Koch's postulates fulfilled, Vet. Q. (1991) 13:131-136.

[27] Thacker E.L., Immunology of the porcine respiratory disease complex, Vet. Clin. North Am. Food Anim. Pract. (2001) 17:551-565.

[28] Van Gucht S., Labarque G., Van Reeth K., The combination of PRRS virus and bacterial endotoxin as a model for multifactorial respiratory disease in pigs, Vet. Immunol. Immunopathol. (2004) 102:165-178.

[29] Vezina S.A., Loemba H., Fournier M., Dea S., Archambault D., Antibody production and blastogenic response in pigs experimentally infected with porcine reproductive and respiratory syndrome virus, Can. J. Vet. Res. (1996) 60:94-99.

[30] Wensvoort G., Terpstra C., Pol J.M., ter Laak E.A., Bloemraad M., de Kluyver E.P., et al., Mystery swine disease in The Netherlands: the isolation of Lelystad virus, Vet. Q. (1991) 13:121-130.

[31] Wieczorek-Krohmer M., Weiland F., Conzelmann K., Kohl D., Visser N., van Woensel P., et al., Porcine reproductive and respiratory syndrome virus (PRRSV): monoclonal antibodies detect common epitopes on two viral proteins of European and U.S. isolates, Vet. Microbiol. (1996) 51:257-266.

[32] Wu W.H., Fang Y., Farwell R., Steffen-Bien M., Rowland R.R., Christopher-Hennings J., Nelson E.A., A $10-\mathrm{kDa}$ structural protein of porcine reproductive and respiratory syndrome virus encoded by ORF2b, Virology (2001) 287:183-191.

[33] Yoon I.J., Joo H.S., Goyal S.M., Molitor T.W., A modified serum neutralization test for the detection of antibody to porcine reproductive and respiratory syndrome virus in swine sera, J. Vet. Diagn. Invest. (1994) 6:289-292.

[34] Yoon K.J., Zimmerman J.J., Swenson S.L., McGinley M.J., Eernisse K.A., Brevik A., et al., Characterization of the humoral immune response to porcine reproductive and respiratory syndrome (PRRS) virus infection, J. Vet. Diagn. Invest. (1995) 7:305-312.

[35] Zuckermann F.A., Garcia E.A., Luque I.D., Christopher-Hennings J., Doster A., Brito M., Osorio F., Assessment of the efficacy of commercial porcine reproductive and respiratory syndrome virus (PRRSV) vaccines based on measurement of serologic response, frequency of gamma-IFN-producing cells and virological parameters of protection upon challenge, Vet. Microbiol. (2007) 123:69-85. 